\title{
Use of conjunctival oxygen monitoring in cardiac patients in the emergency unit
}

\author{
S. HARDING \& S. M. PODOLSKY
}

Department of Emergency Medicine, Albert Einstein Medical Center, Philadelphia, PA, $U S A$

\section{SUMMARY}

Conjunctival oxygen monitoring $\left(\mathrm{PcjO}_{2}\right)$ measures conjunctival tissue organ. It has proved useful in helping to evaluate hypovolemic multiple trauma patients, carotid artery surgery patients, as well as others. Little has been written about its use during medical resuscitations. This report reviews its accident and emergency department use in 13 cardiac arrest/life-threatening cardiac dysrhythmia patients. Nine patients expired and four patients survived. Accident and emergency department $\mathrm{PcjO}_{2}$ values in the group which expired averaged 6.3. Those patients who survived averaged 35.4. Subjects who maintained $\mathrm{PcjO}_{2}$ values below 20 did not survive. Furthermore, $\mathrm{PcjO}_{2}$ values taken within the first 8 min of accident and emergency department resuscitation appear to indicate outcome. Conjunctival oxygen monitoring may be useful in monitoring critically ill cardiac patients in the accident and emergency department.

\section{INTRODUCTION}

Conjunctival oxygen measurement has been used experimentally in a variety of clinical situations including trauma (Abraham et al., 1984a), cardiac arrest (Abraham et al., 1984b), artrial flutter (Podolsky et al., 1985) and carotid artery surgery (Shoemaker \& Lawner, 1983) to delineate its usefulness in the evaluation of critically ill patients. This study reports the results of conjunctival oxymetry in 13 hemodynamically compromised patients, with specific emphasis on the prediction of patient outcome using this monitoring modality.

Correspondence: Dr Sherman M. Podolsky, Department of Emergency Medicine, Albert Einstein Medical Center, York and Tabor Roads, Philadelphia, PA 19141, USA. 


\section{MATERIALS AND METHODS}

All adult patients presenting to the Albert Einstein Medical Center, Northern Division, Emergency Department, Philadelphia, PA, USA between October 1984 and December 1985 who had life-threatening arrhythmias or who were undergoing cardiopulmonary resuscitation were eligible for inclusion in this study, with the following exceptions: patients with glass eyes, conjunctivitis or trauma to the eyes or periorbital areas, and those patients for whom a 'do not resuscitate' status was in effect. Those subjects actually included in the study were those who arrived at the accident and emergency (A\&E) department when one of the authors (S.P.) or his designee was available to initiate conjunctival oxygen monitoring.

Upon arrival to the A\&E department, patients undergoing resuscitation or who appear to be in imminent need of resuscitation are placed in the resuscitation suite, where continuous electrocardiographic monitoring, respiratory and fluid support, surgical equipment and X-ray capability are all readily available. Shortly after their placement in the resuscitation suite, an Orange Medical (Costa Mesa, CA, USA) conjunctival sensor was placed against the right sclera of those patients included in this study. The sensor consists of a miniaturized Clark-type electrode mounted in a ringshaped ophthalmic conformer made of optical grade polymethacrylate (a material used for contact lenses). The current produced at the electrode by the reduction of diffused oxygen is converted to a digital display by a portable electronic monitor which provides a continuous readout of oxygen tension in $\mathrm{mmHg}$. The sensor is calibrated using a two point system, and once it is placed against the palpebral conjunctiva, stabilization is completed within 2 min.

The sensor is placed by first gently retracting the upper eyelid and slipping the uppe part of the conforming ring under the lid, then retracting the lower lid and sliding the lower arm of the ring under that lid. The sensor is then held gently against the palpebral conjunctiva by the pressure of the eyelids. No corneal contact is made, and vision and eye motion are not restricted.

The digital readout was recorded by the investigator every 30-180 s after an initial 2min sensor stabilization period. Cardiac rhythm and interventional measures (e.g. defibrillation and intubation) were also recorded, as was the outcome of the resuscitation itself. Interventions and further laboratory tests were utilized according to the conditions and needs of the patients as determined by the leader of the resuscitation team. Pcj02 measurements were not made available to this person.

Statistical analysis of the data thus obtained was performed using the two-tailed Student's $t$-distribution and the 'four-fold' Chi-square test with Yate's correction. 


\section{RESULTS}

In the course of the study, 13 subjects were studied. Of these, four survived their initial catastrophic process and nine expired. The results contained herein apply only to initial survival; no attempt at long-term follow-up was made.

Of the nine subjects who expired (Group I), eight presented in asystole; the ninth in ventricular fibrillation. The four subjects who survived (Group II) all presented in ventricular tachycardia. Conjunctival oxygen $\left(\mathrm{PcjO}_{2}\right)$ readings in Group I ranged from 0 to 29 (average 6.3) during their first $6 \mathrm{~min}$ of recorded monitoring (actually minutes 2 through 8 , given the 2 min stabilization period for the sensor), while $\mathrm{PcjO}_{2}$ readings in Group II ranged from 18 to 66 (average 35.4 ) in that same time span (see Table 1 ).

Table 1 Presenting cardiac rhythms, patient outcome, and $\mathrm{PcjO}_{2}$ measurements in 13 critically ill patients

\begin{tabular}{lccccc}
\hline & Presenting & & & \multicolumn{2}{c}{$\mathrm{PcjO}_{2}$} \\
\cline { 5 - 6 } \cline { 5 - 6 } Patient & Rhythm & Outcome & & Range & Average \\
\hline Group I & & & & \\
$\# 1$ & Asystolic & Expired & & $8-13$ & $12 \cdot 2$ \\
$\# 2$ & Asystolic & Expired & & $2-29$ & $11 \cdot 6$ \\
$\# 4$ & Asystolic & Expired & & $4-6$ & $4 \cdot 8$ \\
$\# 6$ & Asystolic & Expired & & $1-13$ & $5 \cdot 5$ \\
$\# 8$ & Asystolic & Expired & & $2-12$ & $6 \cdot 2$ \\
$\# 9$ & Asystolic & Expired & & $1-3$ & $1 \cdot 8$ \\
$\# 11$ & VF & Expired & & $0-3$ & $1 \cdot 0$ \\
$\# 12$ & Asystolic & Expired & & $0-0$ & $0 \cdot 0$ \\
$\# 13$ & Asystolic & Expired & & $6-15$ & $8 \cdot 8$ \\
Group II & & & & \\
$\# 3$ & VT & Survived & & $18-40$ & $27 \cdot 5$ \\
$\# 5$ & VT & Survived & $26-38$ & $31 \cdot 3$ \\
$\# 7$ & VT & Survived & $58-66$ & $61 \cdot 7$ \\
$\# 10$ & VT & Survived & $29-33$ & 31.5 \\
\hline
\end{tabular}

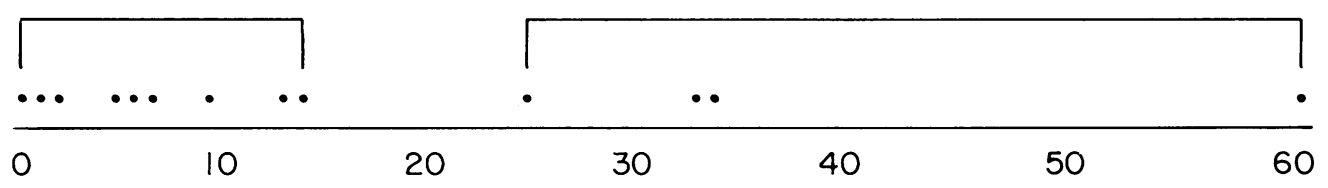

Fig. 1. Average $\mathrm{Pcj}_{2}$ measurements in two groups of critically ill cardiac patients. 
Statistical analysis of the data reveals the following: (1) there is a statistically $\frac{\mathbb{Q}}{0}$ significant difference between the $\mathrm{PcjO}_{2}$ levels reached by Group I and those of Group II (Student's $t$-distribution, $P<0.0001$ ), and (2) subjects who maintain $\mathrm{PcjO}_{2}$ readings below 20 do not survive (Chi-square, four-fold with Yates's correction, $P<0.01$ ) (see Fig. 1). However, one $\mathrm{PcjO}_{2}$ reading below 20 in the first $6 \mathrm{~min}$ of recording has no significance.

\section{DISCUSSION}

This study was undertaken primarily to determine whether or not $\mathrm{PcjO}_{2}$ measurements are useful in ED patients who suffer out-of-hospital cardiac arrest or who present with a life-threatening cardiac dysrhythmia.

As shown in Table 1 , nearly all the subjects in Group I were asystolic upon presentation to the ED. In contrast, all of the subjects in Group II presented in $\omega_{\omega}^{\omega}$ ventricular tachycardia. It has long been noted that asystolic patients respond to resuscitative efforts far less often than do patients in ventricular tachycardia or ventricular fibrillation and (Diamond et al., 1977; Iseri et al., 1977; Myerburg et al., 1980; Smith \& Bodai, 1985) and these data are consistent with this finding.

Also illustrated in Table 1 are the range and average of $\mathrm{PcjO}_{2}$ measurements in subjects in both groups. It is obvious from this table that those subjects who died had

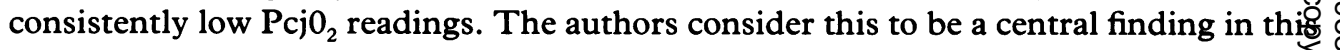
study. In contrast to subjects in Group II who had occasional low readings whick. immediately rose when their arrhythmias were treated, subjects who died maintaine low $\mathrm{PcjO}_{2}$ measurements. The difference between the measurements in Group I and those in Group II is statistically significant, as noted above. This indicated that subjects in Group I came from a population that does not respond to resuscitation, while Group II subjects came from a population that does respond to these efforts. Likewise, the Chi-square test predicts that patients who maintain low $\mathrm{PcjO}_{2}$ measurements will not survive. Figure 1 demonstrates that there was no overlap between the average $\mathrm{PcjO}_{2}$ readings in the two groups.

Survivors were monitored far longer than subjects who died (Group I, av. 16.7 min, Group II, av. $60 \cdot 8 \mathrm{~min}$.) Data was taken from the first $8 \mathrm{~min}$ of monitoring for use in the statistical calculations in this study.

There are other works written relative to our report. In Abraham's study of 11 patients undergoing CPR, it was noted that tissue oxygenation and perfusion changes occurred in patients undergoing CPR, however survival rates in these patients were not discussed (Abraham et al., 1984b). This issue was addressed in our study. In a single case report Abraham \& Ehrlich (1984) did record $\mathrm{PcjO}_{2}$ measurements in a patient whose initial rhythm was asystole and appeared to have a $\mathrm{PcjO}_{2}$ of less than $10 \mathrm{mmHg}$. This patient expired. This was confirmed in the present study (Abraham \& Ehrlich, 1984).

Eliastam et al. (1977) have suggested that patients should have ACLS discontinued if they do not respond to $30 \mathrm{~min}$ or more of ACLS or if they have no ventricular EKG 
activity after more than $10 \mathrm{~min}$ of ACLS. The present authors' mortality data is supportive of that suggestion. Furthermore, this data suggests that the outcome of ACLS can be prognosticated within the first $8 \mathrm{~min}$ of ED resuscitation by the use of conjunctival oxygen monitoring.

It must be reiterated that this study involved resuscitation of patients brought to the ED after suffering an out-of-hospital cardiac arrest or who had life-threatening cardiac dysrhythmias. All data presented from this data are relevant only to this type of patient, and should not be extended to those patients experiencing cardiac arrest while inpatients on wards or in intensive care units. Moreover, this study considered very small groups, and no attempt was made to determine the length of resuscitation time prior to the subjects' arrival in the ED. It is suggested that further study of conjunctival oxygen monitoring with larger groups than this and with attention to the length of resuscitation measures in the out-of-hospital setting would be helpful in the ascertainment of when and for how long ACLS should be employed.

In the current environment of limited physical resources and curtailed budgets, guidelines are needed for allocation of these physical and monetary reserves. The cost of resuscitation attempts varies depending upon the hospital and the lengths to which the rescuers go (e.g. intubation, temporary pacemakers and defibrillation). Obviously, the longer the attempt goes on, the higher its potential cost, not only in money but in terms of availability of physicians and other personnel to care for other-possibly more salvageable-patients.

The present authors have presented data which suggests that $\mathrm{PcjO}_{2}$ measurements within the first $8 \mathrm{~min}$ of ACLS in the ED can indicate the outcome of resuscitation. From this data, they believe that conjunctival oxygen monitoring is a useful additional parameter which, in conjunction with traditional methods, can help to determine when to discontinue resuscitation in a patient who has suffered an out-of-hospital cardiac arrest.

\section{REFERENCES}

Abraham E. \& Ehrlich H. (1984) Conjuctival and transcutaneous oxygen monitoring during resuscitation. Annals of Emergency Medicine 12, 287-9.

Abraham E., Oye R. K. \& Smith M. (1984a) Detection of blood volume deficits through conjunctival oxygen tension monitoring. Critical Care Medicine 12, 931-4.

Abraham E., Smith M. \& Silver L. (1984b) Conjunctival and transcutaneous oxygen monitoring during cardiac arrest and cardiopulmonary resuscitation. Critical Care Medicine 12, 419-421.

Diamond N. J., Schofferman J. \& Elliott J. W. (1977) Factors in successful resuscitation by paramedics. FACEP 6, 42-6.

Eliastam M., Duralde T., Martinez F. \& Schwartz D. (1987) Cardiac arrest in the emergency medical service system: guidelines for resuscitation. $\mathcal{J} A C E P$ 6, 525-9.

Iseri L. T., Siner E. J. \& Humphrey S. B. (1977) Prehospital cardiac arrrest after arrival of the paramedic unit. $\mathcal{F} A C E P$ 6, 530-5.

Myerburg R. J., Conde C. A. \& Sung R. J. (1980) Clinical, electrophysiologic and hemodynamic profile of patients resuscitated from pre-hospital cardiac arrest. American fournal of Medicine 68, 568-76.

Podolsky S. M., Waninger K. \& Wertheimer J. (1985) Conjunctival oxygen monitoring in atrial flutter. American fournal of Emergency Medicine 1, 541-2. 


\section{S. Harding and S. M. Podolsky}

Shoemaker W. C. \& Lawner P. L. (1983) Method for continuous conjunctival oxygen monitoring during carotid artery surgery. Critical Care Medicine 11, 946-7.

Smith J. P. \& Bodai B. I. (1985) Guidelines for discontinuing cardiopulmonary resuscitation in the emergency department after non-paramedic-directed pre-hospital cardiac arrest. Western fournal Medicine 143, 402-5. 